\title{
The Effects of Magnesium-EDTA Chelation Therapy on Arterial Stiffiness
}

\author{
P. J. Van der Schaar ${ }^{1}$, R. Th. B. Pahlplatz ${ }^{2 *}$, E. Blaurock-Busch ${ }^{3 *}$ \\ ${ }^{1}$ International Board of Clinical Metal Toxicology, Leende, The Netherlands \\ ${ }^{2}$ International Biomedical Centre, Leende, The Netherlands \\ ${ }^{3}$ Micro Trace Minerals Laboratory, Hersbruck, Germany \\ Email: pjvanderschaar@gmail.com, ${ }^{*}$ dr.pahlplatz@gmail.com, ${ }^{*}$ ebb@microtrace.de
}

Received 28 September 2014; revised 14 November 2014; accepted 30 November 2014

Copyright (C) 2014 by authors and Scientific Research Publishing Inc.

This work is licensed under the Creative Commons Attribution International License (CC BY). http://creativecommons.org/licenses/by/4.0/

(C) (i) Open Access

\begin{abstract}
Traditional risk factors for cardiovascular disease can only assess risks for groups of people. New parameters of arterial stiffness are more reliable for predicting cardiovascular outcomes for individuals with and without a cardiovascular history. The objective of this study was to assess the effects of Magnesium-EDTA chelation therapy using new methods and parameters such as pulse wave velocity (PWV), central blood pressure (SBPao) and endothelial function (Aix). We followed 43 patients with an abnormal PWV and SBPao, setting them up in two groups. The 21 patients in Group A had already been diagnosed with cardiovascular disease. The other 22 patients in Group B also showed abnormal PWV, SBPao and Aix, but showed no cardiovascular symptoms. Each patient in Groups A and B received one Mg-EDTA treatment per week. The total treatment plan consisted of 25 Mg-EDTA chelation treatments according to the standard protocol of IBCMT. After 25 Mg-EDTA chelation sessions, PWV and SBPao improved significantly in all patients of Groups A and $B$. In addition, Aix improved significantly in these patients, but remained abnormal. Group $C$ included 18 asymptomatic patients with normal PWV or SBPao. Aix was abnormal in this group, but to a much lesser extent than Groups $A$ and $B$. The 18 asymptomatic patients of Group $C$ did not receive Mg-EDTA treatment. Observation showed no significant changes in all three parameters of arterial stiffness. The results of this study indicate that a course of treatment with MagnesiumEDTA chelation therapy significantly lowers cardiovascular risks. We conclude that Mg-EDTA chelation therapy improves PWV as an indicator of arterial stiffness, SBPao (central blood pressure) as an indicator of aortic elasticity and Aix (augmented aortic index) as an indicator of endothelial functioning. These improvements in PWV, SBPao and Aix demonstrate that atherosclerosis is a dynamic and (partially) reversible process.
\end{abstract}

\section{Keywords}

Arterial Stiffness, Pulse Wave Velocity, Central Blood Pressure, Augmented Aortic Index,

${ }^{*}$ Corresponding authors 


\section{Introduction}

Intravenous chelation therapy with Magnesium-EDTA (Mg-EDTA) for cardiovascular disease has been controversial for decades until recently when the randomized double-blind TACT study revealed that treatment with Mg-EDTA reduces risks for cardiac death, hospitalization and invasive cardiac procedures compared to placebo [1]. We wanted to assess how individual cardiovascular risk factors respond to a course of treatments with Magnesium-EDTA according to the Guidelines of the International Board of Clinical Metal Toxicology [2].

\section{Review of Literature}

Standard risk factors for cardiovascular disease have prognostic value for a large population, but little prognostic value for an individual. Although several scoring systems for cardiovascular risk prediction are valuable in the assessment and management of asymptomatic individuals, differences between predicted and actual events do exist. The concept of vascular aging as a cumulative measure of the impact of cardiovascular risk factors on the arterial wall has the potential to assess an individual's overall cardiovascular risk. In this context, candidate arterial biomarkers, apart from proving an incremental predictive value over and above traditional risk factors, must fulfill stringent criteria in order to be integrated into clinical practice. In 2000, L. Terry Chappell et al. concluded that brachial artery stiffness testing appeared to be a good outcome measurement for patients treated with EDTA-chelation therapy [3]. Current evidence suggests that arterial stiffness fulfills the criteria for a biomarker of vascular aging, whereas central hemodynamics and carotid intima-media thickness are close [4].

Arterial stiffness measured as pulse wave velocity (PWV) is highly correlated with coronary atherosclerosis in asymptomatic patients [5]. Pulse wave velocity predicts all-cause mortality in the general population: Participants in the highest PWV-tertile showed an increased risk of all-cause mortality evidenced by a multivariable adjusted hazard ratio of 6.8 (95\% confidence interval: 1.4 - 32.8) as compared with the lowest tertile [6]. Aortic stiffness expressed as aortic PWV is a strong predictor of future cardiovascular events and all-cause mortality. An increase in aortic PWV by $1 \mathrm{~ms}^{-1}$ corresponds to an age-, sex-, and risk factor-adjusted risk increase of $14 \%$, $15 \%$, and $15 \%$ in total cardiovascular events, cardiovascular mortality, and all-cause mortality, respectively. An increase in aortic PWV by 1 standard deviation was associated with respective increases of $47 \%, 47 \%$, and $42 \%$ [7]. PWV predicts cardiovascular incidents better than traditional risk factors including $24 \mathrm{~h}$ blood pressure recording [8]. Russian researchers correlated PWV and coronary stenosis with coronary angiography and showed that a PWV $>12.2 \mathrm{~ms}^{-1}$ is prognostic for a $50 \%$ blockage in coronary arteries [9].

Central pressure has been shown to relate more strongly to vascular disease and outcome than traditional upper arm blood pressure. Central pressure can also distinguish between the effects of different hypertension medications when upper arm blood pressure and pulse wave velocity do not [5].

Up until recently, the effects of Mg-EDTA could only be evaluated by subjectively assessing patient's complaints and by increased walking distance or improved exercise tolerance during stress-testing. The parameters used in the TACT study were also an approximation of what actually happened in the patient's vascular system. Iron (Fe) chelation already has shown to improve endothelial functioning as assessed by the augmented aortic index (Aix) [10]. Mg-EDTA chelates Fe and toxic elements such as lead (Pb) and cadmium (Cd), which have detrimental effects on endothelium and eventually the arterial wall [11].

We established a pilot study to investigate how Mg-EDTA chelation therapy influences these new and more objective cardiovascular risk factors: Aix, SBPao and PWV.

\section{Materials and Methods}

1) Patient selection

We followed 43 patients with an abnormal PWV and Aix. Of these, 21 patients in Group A had already been diagnosed with cardiovascular disease. The other 22 patients in Group B also showed elevated AIX and PWV, but were cardiovascular asymptomatic. Group B was considered at high risk for developing cardiovascular disease. 
All 43 individuals (Groups A and B) were treated 25 times with Mg-EDTA chelation therapy in weekly sessions according to the standard protocol of IBCMT. After 25 chelation sessions the Aix, SBPao and PWV were determined again.

The control group (Group C), consisted of 18 patients with a normal PWV. Aix was abnormal in this group, but to a much lesser extent than Groups A and B. Group C patients were without cardiovascular disease and did not receive Mg-EDTA treatment. It was our aim to find out if risk factors would change in Group C compared to patients with known cardiovascular disease.

2) Testing methods

PWV (arterial stiffness analysis), Aix (endothelial function) and SBPao (central blood pressure) were performed with the Tensiomed ${ }^{\circledR}[10][12]$.

3) Treatment method

3 gr of disodium-EDTA and 5 gr of magnesium sulfate were added to $500 \mathrm{ml}$ of a $0.9 \%$ saline solution along with 1.49 gr potassium chloride, $20 \mathrm{mg}$ thiamine, $100 \mathrm{mg}$ pyridoxine, $0.5 \mathrm{mg}$ hydrocobalamin and 5000 IU heparin. This "Mg-EDTA solution" was infused slowly over 3 hrs according to IBCMT Protocol [2].

Reference ranges: Table 1 and Table 2.

\section{Results}

PWV (aortic stiffness) improved significantly after 25 treatments with Mg-EDTA chelation therapy in all $(\mathrm{N}=$ 43) treated patients (Table 3). The initial PWV was $11.7 \mathrm{~ms}^{-1}$ and normalized $\left(9.0 \mathrm{~ms}^{-1}\right)$ after treatment.

SBPao (central blood pressure) improved significantly after 25 Mg-EDTA chelation therapies in all $(\mathrm{N}=43)$ treated patients from $148.3 \mathrm{~mm} \mathrm{Hg}$ to $131.6 \mathrm{~mm} \mathrm{Hg}$.

Aix (endothelial function) improved significantly after 25 Mg-EDTA chelation therapies in all $(\mathrm{N}=43)$ treated patients, but was still abnormal. It decreased from $26.8 \%$ to $11.5 \%$.

When differentiating between patients with (Group A) and without (Group B) a prior cardiovascular history, we obtained the following results:

Group A

PWV (aortic stiffness) improved significantly after 25 treatments with Mg-EDTA chelation therapy in all (N $=21$ ) treated patients with known cardiovascular disease and with an initially abnormal PWV (Group A; Table 4). The PWV did not normalize completely, but went from risk category IV $\left(12.4 \mathrm{~ms}^{-1}\right.$ abnormal) to category III (10.1 $\mathrm{ms}^{-1}$ increased).

SBPao (central blood pressure) improved significantly after 25 Mg-EDTA chelation therapies in all $(\mathrm{N}=21)$ treated patients with cardiovascular disease from $146.3 \mathrm{~mm} \mathrm{Hg}$ to $133.9 \mathrm{~mm} \mathrm{Hg}$.

Aix (endothelial function) improved significantly after 25 Mg-EDTA chelation therapies in all $(\mathrm{N}=21)$ treated patients with cardiovascular disease, but was still abnormal. It decreased from 31.2\% to $12.2 \%$.

Group B

PWV (aortic stiffness) improved significantly after 25 treatments with Mg-EDTA chelation therapy in all (N = 22) treated patients without prior cardiovascular disease but with an initial, abnormal PWV (Group B; Table 5). The PWV normalized completely from $11.2 \mathrm{~ms}^{-1}$ to $8.4 \mathrm{~ms}^{-1}$.

SBPao (central blood pressure) improved significantly after 25 Mg-EDTA chelation therapies in all $(\mathrm{N}=22)$ treated patients without known cardiovascular disease but with an initially abnormal PWV. SBPao decreased from 148.3 to 129.8 .

Aix (endothelial function) improved significantly after 25 Mg-EDTA chelation therapies in all $(\mathrm{N}=22)$ treated patients with cardiovascular disease. The risk category of the Aix in Group B went from category IV (abnormal) to III (increased). Aix decreased from 21.6\% to 4.4\%.

Control Group C

The control group of 18 asymptomatic patients with a normal PWV (Group C, Table 6) showed no significant changes in all three parameters of arterial stiffness after one year follow-up without intervention. The PWV went from $9.7 \mathrm{~ms}^{-1}$ to $9.5 \mathrm{~ms}^{-1}$. The SBPao changed minimally from $139.9 \mathrm{~mm} \mathrm{Hg}$ to $138.9 \mathrm{~mm} \mathrm{Hg}$. The Aix showed a tendency to improvement from $10.9 \%$ to $3.7 \%$ but without significance $(\mathrm{P}=0.19)$.

\section{Discussion}

Mg-EDTA chelation therapy is a treatment used for cardiovascular disease. Worldwide, physicians are using 
Table 1. Reference ranges of aortic augmentation index (Aix).

\begin{tabular}{cccc}
\hline Risk category & \multicolumn{2}{c}{ Aortic Augmentation Index (Aix) } \\
\hline I & Optimal & Aix $<30 \%$ & No signs of endothelial dysfunction \\
II & Normal & $-30 \%<$ Aix $<-10 \%$ & No signs of endothelial dysfunction \\
III & Increased & $-10 \%<$ Aix $<10 \%$ & Signs of endothelial dysfunction \\
IV & Abnormal & Aix $>10 \%$ & Clearly endothelial dysfunction \\
\hline
\end{tabular}

Table 2. Reference ranges of pulse wave velocity (PWV).

\begin{tabular}{cccc}
\hline Risk category & Puls wave velocity (PWV) & \\
\hline I & Optimal & PWV $<7.0 \mathrm{~ms}^{-1}$ & No signs of aortic stiffness \\
II & Normal & $7.0<\mathrm{PWV}<9.7 \mathrm{~ms}^{-1}$ & No signs of aortic stiffness \\
III & Increased & $9.7<\mathrm{PWV}<12.0 \mathrm{~ms}^{-1}$ & Signs of aortic stiffness \\
IV & Abnormal & $\mathrm{PWV}>12.0 \mathrm{~ms}^{-1}$ & Clear signs of aortic stiffness \\
\hline
\end{tabular}

Table 3. Group A and Group B patients with initial abnormal pulse wave velocity (PWV).

\begin{tabular}{ccccc}
\hline $\mathrm{N}=43$ & Before Mg-EDTA & After Mg-EDTA & Significance & Correlation \\
\hline PWV $\left(\mathrm{ms}^{-1}\right)$ & 11.7 & 9.0 & $<0.001$ & 0.64 \\
SBPao (mm Hg) & 148.3 & 131.6 & $<0.001$ & 0.71 \\
Aix (\%) & 26.8 & 11.5 & $<0.001$ & 0.78 \\
\hline
\end{tabular}

Table 4. Group A: cardiovascular patients with an initial abnormal pulse wave velocity (PWV).

\begin{tabular}{ccccc}
\hline $\mathrm{N}=21$ & Before Mg-EDTA & After Mg-EDTA & Significance & Correlation \\
\hline PWV $\left(\mathrm{ms}^{-1}\right)$ & 12.4 & 10.1 & 0.004 & 0.66 \\
SBPao (mm Hg) & 146.3 & 133.9 & 0.011 & 0.87 \\
Aix (\%) & 31.2 & 12.2 & 0.007 & 0.84 \\
\hline
\end{tabular}

Table 5. Group B: asymptomatic patients with an abnormal pulse wave velocity (PWV).

\begin{tabular}{ccccc}
\hline $\mathrm{N}=22$ & Before Mg-EDTA & After Mg-EDTA & Significance & Correlation \\
\hline PWV $\left(\mathrm{ms}^{-1}\right)$ & 11.2 & 8.4 & $<0.001$ & 0.57 \\
SBPao (mm Hg) & 148.3 & 129.8 & $<0.001$ & 0.74 \\
Aix (\%) & 21.6 & 4.4 & $<0.001$ & 0.70 \\
\hline
\end{tabular}

Table 6. Asymptomatic patients (Group C) with an initial normal pulse wave velocity (PWV).

\begin{tabular}{cccc}
\hline $\mathrm{N}=18$ & First measurement & After 1 year & Significance \\
\hline PWV $\left(\mathrm{ms}^{-1}\right)$ & 9.7 & 9.5 & 0.74 \\
SBPao (mm Hg) & 139.9 & 138.9 & 0.86 \\
Aix (\%) & 10.9 & 3.7 & 0.19 \\
\hline
\end{tabular}

Mg-EDTA for the treatment of cardiovascular disease, and have done so for several decades. In the UK, Mg-EDTA is used since 1985, in the USA since the 1950s.

Most of the research published in the early and middle 1950s, relates to aspects of EDTA's treatment of arterial disease. The most recent study, financed by the National Institute of Health (NIH) was a Trial to Assess Chelation Therapy (TACT). The purpose of this randomized, double blind, placebo-controlled, $2 \times 2$ factorial clinical trial was to assess the benefits and risks of EDTA chelation. TACT followed 1708 patients for an aver- 
age of approximately 4 years. The study was published in 2013, and concluded EDTA's safety and effectiveness for individuals that have been diagnosed with coronary artery disease [1].

According to IBCMT protocol, a course of treatment consists of a series of intravenous infusions of a solution containing the synthetic chelating agent EDTA (Ethylene-Diamin-Tetraacetic Acid) combined with magnesium. Each infusion takes about 3 hours and depending on the patient's condition, infusions are repeated weekly for a series of treatments. In this case, Groups A and B patients each received a total of 25 infusions. Group C did not receive Mg-EDTA.

All Mg-EDTA treated patients with an initially abnormal PWV showed significant improvement of the PWV. In Group A patients (Table 3) with known cardiovascular disease, the PWV decreased by $2.3 \mathrm{~ms}^{-1}$, in Group B (Table 4) without known cardiovascular disease by $2.8 \mathrm{~ms}^{-1}$. There was no statistical difference between Groups A and B, but Group A had initially a higher PWV than Group B. This is interpreted as a logic difference. Group A has already been diagnosed with cardiovascular disease. The pathological situation of the Group A patients may be considered more advanced than that of the Group B patients who have not been diagnosed with cardiovascular disease, yet. Arterial stiffening is more severe in Group A than in Group B.

Mg-EDTA treatment lowered central blood pressure in Groups A and B, which is indicative of better aortic elasticity [13]. The decrease in central blood pressure was more pronounced in Group B, which is another indication that the extent of arterial stiffening was not advanced in this group yet, as it was in Group A. Group B started with a slightly higher central blood pressure compared to Group A, which had been diagnosed with cardiovascular disease and was being treated with antihypertensive medications.

Endothelial function (Aix) improved significantly in Groups A and B. This improvement was clearly more pronounced in Group B, yet another indication that arterial stiffening or in this case, endothelial stiffening, was not as advanced as in Group A. It should be pointed out however, that of the three parameters PWV, SBPao and Aix, endothelial function (Aix) improved the least.

Control Group C, which had not received Mg-EDTA treatments, showed no significant changes in arterial stiffness parameters within one year follow-up.

\section{Conclusions}

Mg-EDTA chelation therapy improves parameters of arterial stiffness: Pulse Wave Velocity as an indicator of arterial stiffness; central blood pressure (SBPao) as an indicator of aortic elasticity and endothelial functioning (augmented aortic index: Aix). These improvements in PWV, SBPao and Aix indicate that atherosclerosis is a dynamic and (partially) reversible process.

This supports the concept that maintenance treatment with Mg-EDTA minimizes risk factors leading to cardiovascular events. As in the TACT trial, we recommend that Magnesium-EDTA treatments are initially administered once a week. Depending on patient symptoms and response, the frequency can gradually be decreased to monthly intervals. In future work we plan to demonstrate how these individual cardiovascular parameters uphold when the frequency of treatment with Magnesium-EDTA has been maintained once a month for at least one year.

\section{Conflict of Interest}

None.

\section{References}

[1] Lamas, G.A., et al. (2013) Effect of Disodium EDTA Chelation Regimen on Cardiovascular Events in Patients with Previous Myocardial Infarction: The TACT Randomized Trial. JAMA, 309, 1241-1250. http://dx.doi.org/10.1001/jama.2013.2107

[2] The International Board of Clinical Metal Toxicology (IBCMT.com).

[3] Chappell, L.T., et al. (2000) Brachial Artery Stiffness Testing as an Outcomes Measurement for EDTA-Treated Patients with Vascular Disease. Clinical Practice of Alternative Medicine, 1, 225-228.

[4] Vlachopoulos, C., et al. (2012) Progress towards Identifying Biomarkers of Vascular Aging for Total Cardiovascular Risk Prediction. Journal of Hypertension, 30, S19-S26. http://dx.doi.org/10.1097/HJH.0b013e328353e534

[5] Liu, C.S., Li, C.I., Shih, C.M., Lin, W.Y., Lin, C.H., Lai, S.W., Li, T.C. and Lin, C.C. (2011) Arterial Stiffness Measured as Pulse Wave Velocity Is Highly Correlated with Coronaryatherosclerosis in Asymptomatic Patients. Journal of 
Atherosclerosis and Thrombosis, 18, 652-658. http://dx.doi.org/10.5551/jat.7021

[6] Turin, T.C., Kita, Y., Rumana, N., Takashima, N., Kadota, A., Matsui, K., Sugihara, H., Morita, Y., Nakamura, Y., Miura, K. and Ueshima, H. (2010) Brachial-Ankle Pulse Wave Velocity Predicts All-Cause Mortality in the General Population: Findings from the Takashima Study. Hypertension Research, 33, 922-925. http://dx.doi.org/10.1038/hr.2010.103

[7] Vlachopoulos, C., Aznaouridis, K. and Stefanadis, C. (2010) Prediction of Cardiovascular Events and All-Cause Mortality with Arterial Stiffness: A Systematic Review and Meta-Analysis. Journal of the American College of Cardiology, 55, 1318-1327. http://dx.doi.org/10.1016/j.jacc.2009.10.061

[8] Roman, M.J., Devereux, R.B., Kizer, J.R., Lee, E.T., Galloway, J.M., Ali, T., Umans, J.G. and Howard, B.V. (2007) Central Pressure More Strongly Relates to Vascular Disease and Outcome than Does Brachial Pressure: The Strong Heart Study. Hypertension, 50, 197-203. http://dx.doi.org/10.1161/HYPERTENSIONAHA.107.089078

[9] Orlova, I.A., Kuz'mina, A.E., Barinova, I.V., Iarovaia, E.B. and Ageev, F.T. (2009) Assessment of Major Artery Stiffness: New Perspectives of Non-Invasive Diagnosis of Coronary Atherosclerosis. Terapevticheskii Arkhiv, 81, 8-13.

[10] Verma, et al. (2002) Fundamentals of Endothelial Function, Interventions to Improve Endothelial Function. Circulation, 105, 546-549. http://dx.doi.org/10.1161/hc0502.104540

[11] Tellez-Plaza, M., Jones, M.R., Dominguez-Lucas, A., Guallar, E. and Navas-Acien, A. (2013) Cadmium Exposure and Clinical Cardiovascular Disease: A Systematic Review. Current Atherosclerosis Reports, 15, 356. http://dx.doi.org/10.1007/s11883-013-0356-2

[12] Horváth, I.G., Németh, A., Lenkey, Z., Alessandri, N., Tufano, F., Kis, P., Gaszner, B. and Cziráki, A. (2010) Invasive Validation of a New Oscillometric Device (Arteriograph) for Measuring Augmentation Index, Centralblood Pressure and Aortic Pulse Wave Velocity. Journal of Hypertension, 28, 2068-2075. http://dx.doi.org/10.1097/HJH.0b013e32833c8a1a

[13] Roman, M.J., Devereux, R.B., Kizer, J.R., Lee, E.T., Galloway, J.M., Ali, T., Umans, J.G. and Howard, B.V. (2007) Central Pressure More Strongly Relates to Vascular Disease and Outcome than Does Brachial Pressure: The Strong Heart Study. Hypertension, 50, 197-203. http://dx.doi.org/10.1161/HYPERTENSIONAHA.107.089078 
Scientific Research Publishing (SCIRP) is one of the largest Open Access journal publishers. It is currently publishing more than 200 open access, online, peer-reviewed journals covering a wide range of academic disciplines. SCIRP serves the worldwide academic communities and contributes to the progress and application of science with its publication.

Other selected journals from SCIRP are listed as below. Submit your manuscript to us via either submit@scirp.org or Online Submission Portal.
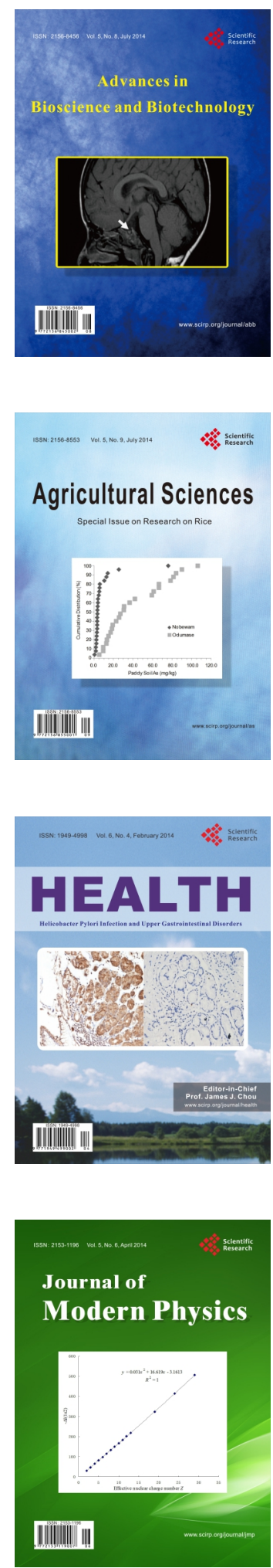
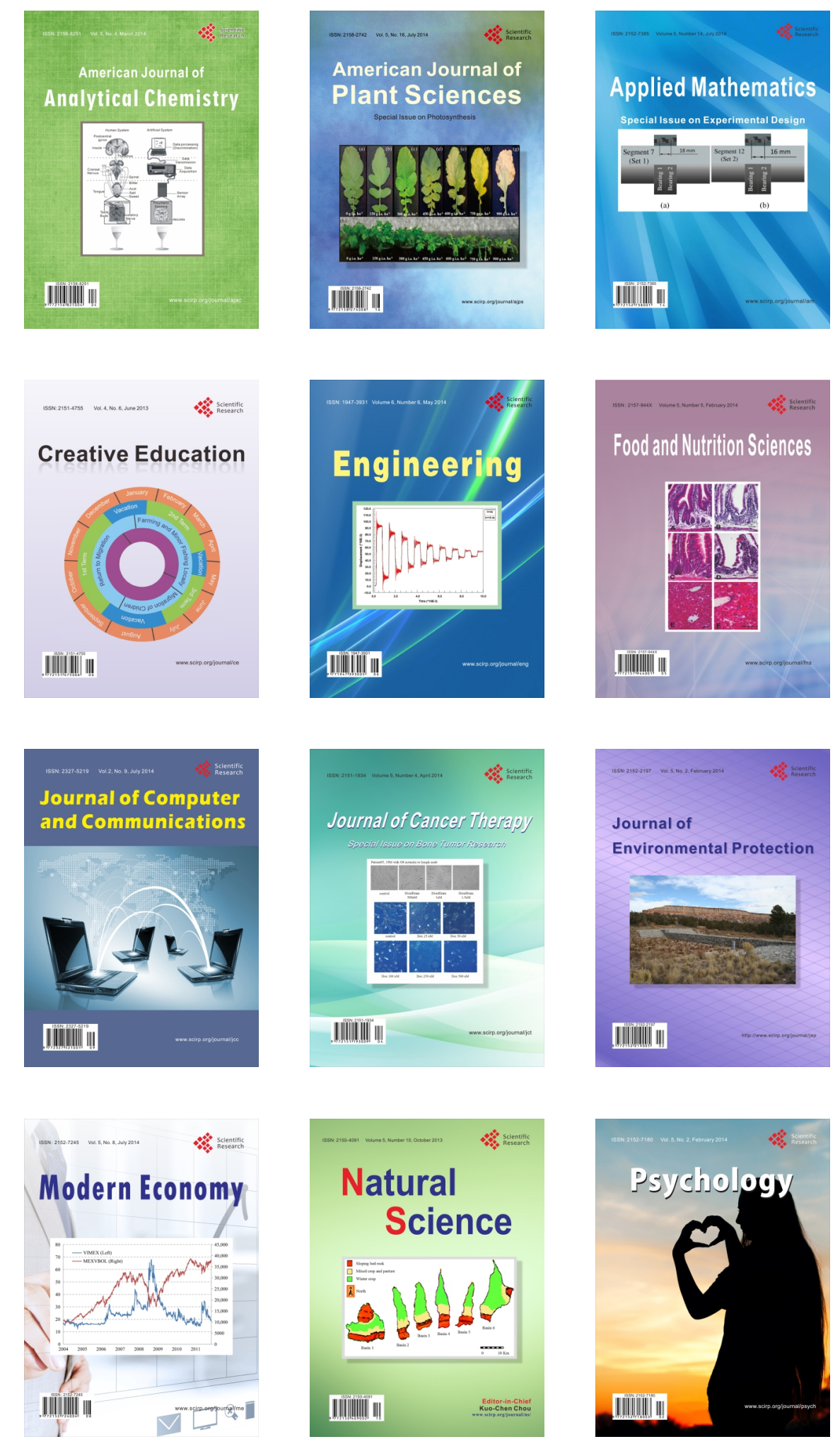